\title{
Many-Channel Landauer-Like Conductance Formula in a Uniform Magnetic Field
}

\author{
F. BenAmira ${ }^{a}$ AND A. AbDellaOUi ${ }^{a, b}$ \\ ${ }^{a}$ Laboratoire de Physique Théorique, Université Mentouri-Constantine, Constantine 25000, Algeria \\ ${ }^{b}$ Département de Physique, Faculté des Sciences Exactes et des Sciences de la Vie et de la Nature \\ Université Larbi Ben M’Hidi, Oum El Bouaghi 04200, Algeria
}

(Received August 22, 2011; in final form June 11, 2012)

\begin{abstract}
Linear-response theory is combined with the Landauer viewpoint to describe quantitatively transport in a four-lead mesoscopic structure within the presence of a uniform magnetic field. A new multichannel magnetoconductance formula is derived in the case where the magnetic field is perpendicular to the current-flow. The invariance under magnetic reversal test is confirmed.
\end{abstract}

PACS: $73.23 .-\mathrm{b}$

\section{Introduction}

For long time, it has been well recognized that transport in mesoscopic systems may relate to their scattering properties as it was originally proposed by Landauer $[1,2]$. The single-channel conductance formula has been first obtained heuristically as proportional to the ratio between transmission and reflection coefficients $G=\frac{2 e^{2}}{h} \frac{T}{R}[1]$.

Main attempts to derive this formula from linear-response theory (LRT) are known in the literature (see Ref. [3] and references therein). However, for long time, the generalization to a multichannel formula has been a great challenge for the physicists. The major difficulty is that, in the LRT, the current-flow in a sample is obtained as a response to an applied external perturbation inside the reservoirs in accordance to the Kubo viewpoint (KVP), whereas in the Landauer viewpoint (LVP) the current itself is considered as responsible for the induction of a position-dependent potential inside the sample. At this level, one has to note that it has been established heuristically that contacts between the reservoirs and the sample are at the origin of the difference between the two points of view [4].

In recent works [5], a theory of transport that combines the LVP with the LRT and which does not consider the effect of the contacts (called thereafter a self-consistent LRT) in order to calculate the relevant part of the density-operator for mesoscopic samples in the absence of an external magnetic field, has been proposed. The obtained density-operator, necessary to evaluate all physical quantities, was used to derive a new and more plausible four-lead multichannel conductance formula that does not endure any of the inconsistencies cited in the literature for the other known formulae $[3,6]$. This self-consistent LRT was recently extended to take into account the existence of a uniform magnetic field $[7,8]$. It has been shown that the density-operator may be written as a sum of two terms [7]: the first represents the Kubo density-operator $\rho^{\mathrm{K}}[7-9]$ and the second defines the contribution of self-consistent effects $\rho^{\mathrm{SC}}[7,8]$ :

$$
\rho^{\mathrm{L}}=\rho^{\mathrm{K}}+\rho^{\mathrm{SC}} \text {. }
$$

Our aim in this work is the use of Eq. (1) to determine quantitatively the magnetoconductance of a four-lead mesoscopic measurement in terms of the scattering matrix elements whenever self-consistent effects are relevant; that is, whenever the effect of the contacts is neglected and the induced potential inside the sample is considered.

\section{Magnetoconductance in terms of $S$ matrix elements}

Consider a mesoscopic scatterer connected via two straight identical semi-infinite perfect leads to two large particle-reservoirs acting as current source and sink, and maintained at constant chemical potentials $\mu_{\mathrm{L}}$ and $\mu_{\mathrm{R}}$, respectively, where $\mu_{\mathrm{L}}-\mu_{\mathrm{R}}$ is small enough to ensure a linear-transport regime. The sample (the mesoscopic disordered part plus the leads without including the contacts) having a volume $\Omega=L S$ is immersed in a uniform perpendicular time-independent weak magnetic field $\boldsymbol{B}=B \hat{z}$

The transport throughout the sample is completely coherent and the measuring probes are ideally coupled in order not to disturb the measurement, see Fig. 1.

In LVP as a response to current-flow between the reservoirs, a position-dependent potential $w(\boldsymbol{r})$ builds up inside the sample due to charges accumulation on both sides of the scatterer $[1,2,10]$. Once the steady-state regime (SSR) is established, $w(\boldsymbol{r})$ will take constant values, $w_{\mathrm{L}}$ and $w_{\mathrm{R}}$, in the left and right asymptotic regions, that are different from the chemical potentials of the adjacent reservoirs; i.e., $w_{\mathrm{L}} \neq \mu_{\mathrm{L}}$ and $w_{\mathrm{R}} \neq \mu_{\mathrm{R}}$, respectively. 


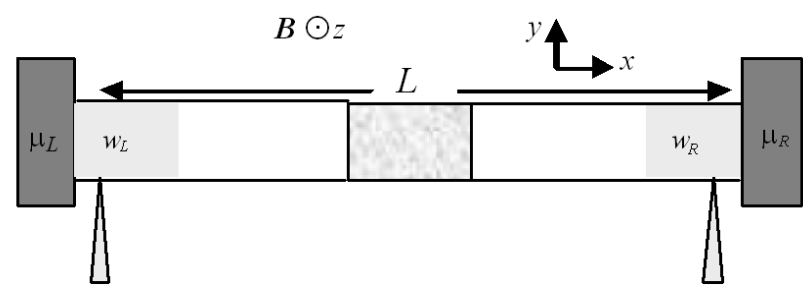

Fig. 1. Mesoscopic sample in a perpendicular magnetic field, with the assistance of non-invasive measuring probes.

The one-particle Hamiltonian for transport carriers in the SSR is then given by

$$
H^{\prime}=H+w(\boldsymbol{r})=H_{0}+V(\boldsymbol{r})+w(\boldsymbol{r}),
$$

where $w(\boldsymbol{r})$ represents the built-up position-dependent potential inside the sample resulting from the self-consistent pile-up of carriers between left and right scatterer sides [1, 2, 5, 11-13], $V(\boldsymbol{r})$ is the electron-impurities interaction potential, and $H_{0}$ is the Hamiltonian of the non-interacting confined electron. In the effective mass approximation $H_{0}=\frac{1}{2 m^{*}}(\boldsymbol{p}-e \boldsymbol{A})^{2}+V_{\mathrm{c}}(\boldsymbol{r})$, where $\boldsymbol{p}$ stands for the momentum operator, $\boldsymbol{A}$ for the vector potential and $V_{\mathrm{c}}(\boldsymbol{r})$ for the confinement potential in the $y$ and $z$ directions. For later use, we choose the Landau gauge: $\boldsymbol{A}=-B y \boldsymbol{i}$.

The eigenfunctions of $H_{0}$ are a product of plane waves propagating in the longitudinal $x$-direction and confined functions in the $y$ and $z$ directions

$$
\varphi_{\xi}(\boldsymbol{r})=\frac{1}{\sqrt{L}} \exp \left( \pm \mathrm{i} k_{a} x\right) \chi_{\xi}(y, z),
$$

where $\xi$ denotes the transmitted channels $a$, the corresponding positive longitudinal wave vector $k_{a}$ and the direction of propagation $\sigma= \pm$; i.e., $\xi \equiv\left(a, k_{a}, \sigma\right)$.

To each of these eigenfunctions with energy eigenvalue $\varepsilon_{\xi}$, corresponds an outgoing $\psi_{\xi}^{+}(\boldsymbol{r})$ and an ingoing $\psi_{\xi}^{-}(\boldsymbol{r})$ scattering states, as the eigenstates of $H$ with the same eigenvalue $\varepsilon_{\xi}[5,9]$. In the asymptotic regions of the leads, at a given energy, $\psi_{\xi}^{ \pm}(\boldsymbol{r})$ are simple combinations of $\varphi_{\xi}(\boldsymbol{r})$; they relate via the left/right transmission and reflection coefficients $t^{\mathrm{L}}, t^{\mathrm{R}}, r^{\mathrm{L}}, r^{\mathrm{R}}$ that define the unitary scattering matrix $S[5,9]$.

\subsection{Current-flow}

Since the sample is not invariant by translation, in the presence of a magnetic field the one-particle current-density operator at point $\boldsymbol{r}^{\prime}$ may be written in a symmetrical form as

$$
\begin{aligned}
& \boldsymbol{j}\left(\boldsymbol{r}^{\prime}\right)=\frac{e}{2 m *}\left[(\boldsymbol{p}-e \boldsymbol{A}) \delta\left(\boldsymbol{r}-\boldsymbol{r}^{\prime}\right)\right. \\
& \left.\quad+\delta\left(\boldsymbol{r}-\boldsymbol{r}^{\prime}\right)(\boldsymbol{p}-e \boldsymbol{A})\right] .
\end{aligned}
$$

In the LVP, the resulting expectation value of the current-flow operator through an arbitrary cross-section $\boldsymbol{S}^{\prime}(i=$ $\int_{\boldsymbol{S}^{\prime}} \mathrm{d} \boldsymbol{S}^{\prime} \cdot \boldsymbol{j}\left(\boldsymbol{r}^{\prime}\right)$ with $\boldsymbol{S}^{\prime}$ may be chosen in the asymptotic regions of the leads) is defined by the trace of the product of $\rho^{\mathrm{L}}$, Eq. (1), by $i$ and may conveniently be separated into a sum of two terms

$$
I=\operatorname{Tr} \rho^{\mathrm{L}} i=I^{\mathrm{K}}+I^{\mathrm{SC}} .
$$

\subsubsection{Kubo's term}

Making use of the $\rho^{\mathrm{K}}$ expression derived in [7, 8], one can easily see that $I^{\mathrm{K}}$ coincides with the expression given by KVP [9], yet now the chemical potentials of the reservoirs are replaced by the induced electrochemical potentials in the asymptotic regions of the leads

$$
I^{\mathrm{K}}=\frac{\Delta w}{2} \int \mathrm{d} \varepsilon\left[-f^{\prime}(\varepsilon)\right] \sum_{\xi} \delta\left(\varepsilon-\varepsilon_{\xi}\right)\left(i^{+}\right)_{\xi \xi},
$$

where $\Delta w=w_{\mathrm{L}}-w_{\mathrm{R}}, f^{\prime}(\varepsilon)$ defines the derivative of the Fermi-Dirac distribution function at energy $\varepsilon$ and $\left(i^{+}\right)_{\xi \xi}=\left\langle\psi_{\xi}^{+}|i| \psi_{\xi}^{+}\right\rangle$is the current-flow matrix element in the outgoing scattering states. The sum over $\xi$ in the right hand side (rhs) of Eq. (5) which reads $\sum_{\xi} \equiv \sum_{a, \sigma} \sum_{k_{a}}$ where $\sum_{k_{a}}$ may be written as $\sum_{k_{a}} \rightarrow_{\mathrm{L} \rightarrow \infty} \frac{L}{h} \int_{\varepsilon_{\xi}}^{\infty} \frac{1}{v_{a}^{\varepsilon_{\xi}}} \mathrm{d} \varepsilon_{\xi}$, with $v_{a}^{\varepsilon_{\xi}}$ representing a $\boldsymbol{B}$ -dependent channel velocity in the $x$-direction at given energy $\varepsilon_{\xi}[9,14]$, is restricted to states of fixed energy $\varepsilon_{\xi}=\varepsilon$ because of the Dirac $\delta$-function. In that case, carrying out the integration over $\varepsilon_{\xi}$, Eq. (5) may be expressed like

$$
I^{\mathrm{K}}=\frac{e \Delta w}{2 h} \int \mathrm{d} \varepsilon\left[-f^{\prime}(\varepsilon)\right] \sum_{a, \sigma}^{\varepsilon} \sigma\left(\boldsymbol{\Pi}^{+}\right)_{a \sigma}^{\varepsilon},
$$

where $\boldsymbol{\Pi}^{+}$is a $2 n$-column vector given by its components $\left(\boldsymbol{\Pi}^{+}\right)_{a \sigma}^{\varepsilon}=\left\langle\bar{\psi}_{a \sigma}^{+, \varepsilon}|i| \bar{\psi}_{a \sigma}^{+, \varepsilon}\right\rangle$ with $\left|\bar{\psi}_{a \sigma}^{+, \varepsilon}\right\rangle=\sqrt{L / v_{a}^{\varepsilon}}\left|\psi_{a \sigma}^{+, \varepsilon}\right\rangle$ are, at a given energy $\varepsilon$, the outgoing scattering states normalized to unit incoming flux: $\left(\boldsymbol{\Pi}^{+}\right)_{(a+)}^{\varepsilon}=-\left(\boldsymbol{t}^{\mathrm{L}+} \boldsymbol{t}^{\mathrm{L}}\right)_{a a}$; $\left(\boldsymbol{\Pi}^{+}\right)_{(a-)}^{\varepsilon}=-\left(\boldsymbol{t}^{\mathrm{R}+} \boldsymbol{t}^{\mathrm{R}}\right)_{a a}$

\subsubsection{Self-consistent term}

In Ref. [7], it has been shown that $\rho^{\mathrm{SC}}=$ $\sum_{\xi}\left|\psi_{\xi}^{+}\right\rangle\left\langle\psi_{\xi}^{+}\right|\left(\rho_{d}^{\mathrm{L}}\right)_{\xi \xi}$, where $\left(\rho_{d}^{\mathrm{L}}\right)_{\xi \xi}=\left\langle\varphi_{\xi}\left|\rho_{d}^{\mathrm{L}}\right| \varphi_{\xi}\right\rangle$ are the matrix elements of the diagonal part of the density-operator $\rho^{\mathrm{L}}$ in the basis of $H_{0}$. Consequently, $I^{\mathrm{SC}}$ may be written as

$$
\begin{aligned}
I^{\mathrm{SC}} & =\sum_{\xi}\left(i^{+}\right)_{\xi \xi}\left(\rho_{d}^{\mathrm{L}}\right)_{\xi \xi} \\
& =\int \mathrm{d} \varepsilon \sum_{\xi} \delta\left(\varepsilon-\varepsilon_{\xi}\right)\left(i^{+}\right)_{\xi \xi}\left(\rho_{d}^{\mathrm{L}}\right)_{\xi \xi},
\end{aligned}
$$

using the ansatz of the $\delta$-function integration on the energy.

Carrying out the sum over $\xi$, while following the same steps leading to Eq. (6), one obtains

$$
I^{\mathrm{SC}}=\frac{e}{h} \int \mathrm{d} \varepsilon \sum_{a, \sigma}\left(\boldsymbol{\Pi}^{+}\right)_{a \sigma}^{\varepsilon}\left(\rho_{d}^{\mathrm{L}}\right)_{a \sigma}^{\varepsilon},
$$

where, as has been shown in $[7,8], \quad\left(\rho_{d}^{\mathrm{L}}\right)_{a \sigma}^{\varepsilon}=$ $\Delta w\left[-f^{\prime}(\varepsilon)\right] \sum_{b \sigma^{\prime}}\left(\overline{\boldsymbol{\Gamma}}^{-1}\right)_{a \sigma \mid b \sigma^{\prime}}^{\varepsilon}\left(\boldsymbol{\Pi}^{-}\right)_{b \sigma^{\prime}}^{\varepsilon}$, where $\bar{\Gamma}^{-1}$ represents the matrix inverse of the matrix $\boldsymbol{\Gamma}$ whose elements are $(\boldsymbol{\Gamma})_{a+\mid b+}^{\varepsilon}=\delta_{a b}-\left|t_{a b}^{\mathrm{L}}\right|^{2},(\boldsymbol{\Gamma})_{a-\mid b-}^{\varepsilon}=\delta_{a b}-\left|t_{a b}^{\mathrm{R}}\right|^{2}$, $(\boldsymbol{\Gamma})_{a+\mid b-}^{\varepsilon}=-\left|r_{a b}^{\mathrm{L}}\right|^{2},(\boldsymbol{\Gamma})_{a-\mid b+}^{\varepsilon}=-\left|r_{a b}^{\mathrm{R}}\right|^{2}$ and $\boldsymbol{\Pi}^{-}$defines a column vector given by its components $\left(\boldsymbol{\Pi}^{-}\right)_{a \sigma}^{\varepsilon}=$ 
$\left\langle\bar{\psi}_{a \sigma}^{-, \varepsilon}|i| \bar{\psi}_{a \sigma}^{-, \varepsilon}\right\rangle$, where $\left|\bar{\psi}_{a \sigma}^{-, \varepsilon}\right\rangle=\sqrt{L / v_{a}}\left|\psi_{a \sigma}^{-, \varepsilon}\right\rangle$ are at a given energy $\varepsilon$ the ingoing scattering states normalized to unit incoming flux $(\boldsymbol{\Gamma})_{a-\mid b-}^{\varepsilon}=\delta_{a b}-\left|t_{a b}^{\mathrm{R}}\right|^{2}\left(\boldsymbol{\Pi}^{-}\right)_{(a+)}^{\varepsilon}=$ $\left(\boldsymbol{t}^{\mathrm{L}} \boldsymbol{t}^{\mathrm{L}+}\right)_{a a} ;\left(\boldsymbol{\Pi}^{-}\right)_{(a-)}^{\varepsilon}=-\left(\boldsymbol{t}^{\mathrm{R}} \boldsymbol{t}^{\mathrm{R}+}\right)_{a a}$ (Refs. [7, 8]).

Otherwise, $I^{\mathrm{SC}}$ may be written in a more explicit form, as

$$
\begin{aligned}
& I^{\mathrm{SC}}=\frac{e \Delta w}{h} \int \mathrm{d} \varepsilon\left[-f^{\prime}(\varepsilon)\right] \\
& \quad \times \sum_{a, \sigma}^{\varepsilon} \sum_{b, \sigma^{\prime}}^{\varepsilon}\left(\boldsymbol{\Pi}^{+}\right)_{a \sigma}^{\varepsilon}\left(\overline{\boldsymbol{\Gamma}}^{-1}\right)_{a \sigma \mid b \sigma^{\prime}}^{\varepsilon}\left(\boldsymbol{\Pi}^{-}\right)_{b \sigma^{\prime}}^{\varepsilon} .
\end{aligned}
$$

At this level, let us mention that in the limit of weak transmission, the $\rho_{d}^{\mathrm{L}}$ matrix elements and thus $\rho^{\mathrm{SC}}$ are negligible to $\rho^{\mathrm{K}}$. Consequently, the self-consistent term, Eq. (9), may be neglected such that $I$ and $I^{\mathrm{K}}$ will lead approximately to the same conductance formula (see below).

\subsection{The magnetoconductance}

Defined as the ratio of the applied current $I$, Eq. (4), to the induced potential difference inside the leads $\Delta w / e$, the resulting magnetoconductance may easily be written

$$
G^{\mathrm{L}}(\boldsymbol{B})=G^{\mathrm{K}}(\boldsymbol{B})+G^{\mathrm{SC}}(\boldsymbol{B}) .
$$

$G^{\mathrm{K}}(\boldsymbol{B})$, the conductance resulting from $I^{\mathrm{K}}$, is given by the Landauer-Büttiker formula $[9,13-15]$ :

$$
G^{\mathrm{K}}(\boldsymbol{B})=\frac{e^{2}}{2 h} \int \mathrm{d} \varepsilon\left[-f^{\prime}(\varepsilon)\right]\left(\boldsymbol{\Pi}^{+}\right)^{t} \boldsymbol{I}
$$

with $t$ denoting the transpose and $\boldsymbol{I}$ is a $2 n$-column vector defined by its components

$$
(\boldsymbol{I})^{t}=\overbrace{\overbrace{+1+1 \ldots+1 \mid-1-1 \ldots-1}^{+1 \ldots}}^{.}
$$

However, making use of Eq. (9), the resulting conductance $G^{\mathrm{SC}}(\boldsymbol{B})$ may also be expressed as an integral over the energy

$$
G^{\mathrm{SC}}(\boldsymbol{B})=\frac{e^{2}}{h} \int \mathrm{d} \varepsilon\left[-f^{\prime}(\varepsilon)\right]\left(\boldsymbol{\Pi}^{+}\right)^{t}\left(\overline{\boldsymbol{\Gamma}}^{-1} \boldsymbol{\Pi}^{-}\right) .
$$

Combining Eqs. (10), (11) and (12) and taking account of the current conservation constraints $[5,9]$, we show after some algebraic manipulations that the many-channel magnetoconductance which excludes the contacts may be put in a more compact form as

$$
G^{\mathrm{L}}(\boldsymbol{B})=2 \frac{e^{2}}{h} \int \mathrm{d} \varepsilon\left[-f^{\prime}(\varepsilon)\right]\left(\boldsymbol{\Pi}^{+}\right)^{t}\left(\overline{\boldsymbol{\Gamma}}^{-1} \boldsymbol{I}\right),
$$

where 2 stands for the spin degeneracy.

This is the sought formula of this work. At first sight, we obtain a magnetoconductance formula which is explicitly independent from channel velocities. Moreover, since the obtained formula is an implicit function on the magnetic field, we can show that this formula remains the same even in a zero magnetic field [8].

\subsubsection{Uncorrelated channels}

Alternatively, treating the case of uncorrelated channels with $\left|t_{a b}^{\mathrm{L}(\mathrm{R})}\right|^{2}=T_{a} \delta_{a b},\left|r_{a b}^{\mathrm{L}(\mathrm{R})}\right|^{2}=R_{a} \delta_{a b}$ it follows after some algebraic manipulations that Eq. (13) may be reduced to a sum of $n$ single-channel Landauer formula

$$
G^{\mathrm{L}}(\boldsymbol{B})=2 \frac{e^{2}}{h} \int \mathrm{d} \varepsilon\left[-f^{\prime}(\varepsilon)\right] \sum_{a}^{\varepsilon} \frac{T_{a}}{R_{a}} .
$$

As it is apparent, in the limit of a ballistic conductor this formula gives an infinite conductance while the Kubo formula defined by Eq. (11) gives a finite conductance.

Moreover, this formula is what we will obtain experimentally in four-lead geometry when the probes measuring the induced voltage in the leads are ideally coupled to the system.

\subsubsection{Magnetic field reversal test}

As a consequence to the unitarity of the scattering matrix $S[5,9]$, Eq. (13) may be expressed like

$$
\begin{aligned}
& G^{\mathrm{L}}(\boldsymbol{B})=\frac{e^{2}}{h} \int \mathrm{d} \varepsilon \\
& \quad \times\left[-f^{\prime}(\varepsilon)\right]\left[\left(\boldsymbol{\Pi}^{+}\right)^{t}\left(\overline{\boldsymbol{\Gamma}}^{-1} \boldsymbol{I}\right)+\left(\boldsymbol{\Pi}^{-}\right)^{t}\left(\overline{\boldsymbol{\Gamma}}^{-1}\right)^{t} \boldsymbol{I}\right] .
\end{aligned}
$$

However, since by reversing the magnetic field the scattering matrix transposes $S(\boldsymbol{B})=S^{t}(-\boldsymbol{B})$, its matrix elements obey to

$$
r_{a a}^{\mathrm{L}(\mathrm{R})}(\boldsymbol{B})=r_{a a}^{\mathrm{L}(\mathrm{R})}(-\boldsymbol{B}) \text { and } t_{a b}^{\mathrm{L}(\mathrm{R})}(\boldsymbol{B})=t_{b a}^{\mathrm{R}(\mathrm{L})}(-\boldsymbol{B}),(16)
$$
we can check that $G^{\mathrm{L}}(\boldsymbol{B})$ is in agreement with the Onsager symmetry relations which assert that transport coefficients are invariant under magnetic field reversal

$$
G^{\mathrm{L}}(\boldsymbol{B})=G^{\mathrm{L}}(-\boldsymbol{B}) .
$$

\section{Conclusion}

Performing a self-consistent LRT in the presence of an external magnetic field, we derived a multichannel magnetoconductance formula that excludes the effect of the contacts and reduces in the limiting case of one channel at zero temperature to the well-known Landauer conductance formula. Moreover, we have shown that this multichannel formula is, as it should be, well consistent with the principle of microscopic reversibility.

\section{Acknowledgments}

The authors thank the referee for his suggestions and recommendations. This work has been sponsored by MESRS and DGRSDT Algeria.

\section{References}

[1] R. Landauer, IBM J. Res. Dev. 1, 223 (1957).

[2] R. Landauer, Philos. Mag. 21, 863 (1970).

[3] A.D. Stone, A. Szafer, IBM J. Res. Develop. 32, 384 (1988).

[4] Y. Imry, in: Perspectives on Condensed Matter Physics, Eds. G. Grinstein, E. Mazenko, World Sci., Singapore 1986, p. 101; Y. Imry, Introduction to Mesoscopic Physics, Oxford University, New York 1997.

[5] F. Benamira, Ph.D. Thesis, Université Montréal, Canada 1996.

[6] M. Büttiker, Y. Imry, R. Landauer, S. Pinhas, Phys. Rev. B 31, 6207 (1985). 
[7] A. Abdellaoui, F. Benamira, Phys. Status Solidi C 1, 3769 (2004).

[8] A. Abdellaoui, Ph.D. Thesis, Constantine, Algeria 2006.

[9] H.U. Baranger, A.D. Stone, Phys. Rev. B 40, 8169 (1989).

[10] S. Datta, Electronic Transport in Mesoscopic Systems, Cambridge University Press, Cambridge 1995.

[11] D.C. Langreth, E. Abrahamas, Phys. Rev. B 24, 2978 (1981).
[12] Y. Imry, R. Landauer, Rev. Mod. Phys. 71, S306 (1999).

[13] J.U. Nöckel, A.D. Stone, H.U. Baranger, Phys. Rev. B 48, 17569 (1993).

[14] K. Shepard, Phys. Rev. B 43, 11623 (1991).

[15] T. Dittrich, P. Hainggi, G.-L. Ingold, B. Kramer, G. Schon, W. Zwerger, Quantum Transport and Dissipation, Wiley-VCH, Weinheim 1998. 